\title{
Thermodynamic Effect of Pressure on Nucleation Activation Energy
}

\author{
Diego Vilar da Silva ${ }^{a *}$ (1), Rodinei Medeiros Gomes ${ }^{a}$ \\ ${ }^{a}$ Universidade Federal da Paraíba (UFPB), Programa de Pós-Graduação em Engenharia Mecânica, \\ 58051-900, João Pessoa, PB, Brasil.
}

Received: December 26, 2020; Revised: May 02, 2021; Accepted: June 04, 2021

\begin{abstract}
The first stage of the solidification of metal alloys is nucleation, during this stage is defined the number of grains present in the final structure, just as the conditions in which the same will grow. The application of pressure in the solidification is mostly studied as an operational parameter, and its effects are described and commented extensively in literature. The objective of this paper is to study the effect of pressure on nucleation, still not to describe but to explain it, for this purpose the pressure is treated as a thermodynamic variable. From this analysis it was possible to determine the real influence of pressure on the activation energy, being clear that its effect is equivalent and co-dependent to that of the undercooling, and can thus cause considerable variations in the activation energy, much larger than what is proposed in previously published papers that approach the subject, causing a considerable stimulus on nucleation. It was also observed that the curve that represents the effect of pressure on the activation energy proposed in this paper, has a similar form to the curves that describe how the pressure refines structures, resulting from the process of solidification under pressure reported in literature.
\end{abstract}

Keywords: Solidification, pressure, nucleation, activation energy, thermodynamics.

\section{Introduction}

Casting is one of the main manufacturing processes used in the production of metal parts, and the effects of the solidification process on these parts is most evident when casting is the final operation. This influence can also be seen in the finished product even after extensive machining, since the solidification structure and its associated defects are difficult to eliminate once they are created, so a good control of the solidification process from the initial stages is of great importance. Nucleation is the dominant process at the beginning of solidification, and it leads quickly to the determination of the final number of grains with each nucleus forming a grain. The conditions that lead to nucleation are therefore of the utmost importance in determining the characteristics of any microstructure ${ }^{1}$.

The mechanical properties of a metal alloy have a strong dependence on the structure produced during solidification, both on the macro and micro scale, and what controls the structural parameters are the thermal variables and the process dynamics, which in practice are controlled by the composition of the alloy, and by operational parameters such as mold preheating temperature, casting temperature, mold geometry, cooling mechanism and others. Each of these operational parameters changes the way the system evolves to its final state in a different fashion, causing variations in the structure of the resulting alloy.

Another operational parameter used in casting is the application of external pressure, according to Sobczak et al. ${ }^{2}$ the application of pressure promotes a more intimate contact between the metal and the mold walls, which causes an

*e-mail: diegovilartpb@hotmail.com increase of the solidification and cooling rates, it also causes a variation in the melting temperature during the solidification of the alloy, among other changes in the properties of the material. In this way the application of pressure during solidification, if used correctly, can refine structures on both macro and micro scales of various metal alloys, thus improving the mechanical properties such as hardness and strength. These attributes make solidification under pressure a process capable of producing high quality components for critical weight applications, such as the automotive industry.

Conventional casting technologies that use pressure are usually based on purely empirical knowledge, and do not consider models originated from the understanding of the physical changes caused by pressure. The understanding of all the complex physical phenomena that accompanies the solidification under pressure is facilitated by the thermodynamic and kinetic descriptions ${ }^{2}$.

In this paper the effect of pressure on nucleation is discussed and explored, but not as an operational parameter but as a thermodynamic variable. When calculating its effects on the physical properties involved in the nucleation process, a set of equations was obtained and its implications were compared with the results published in the literature, making it clear that the effect of pressure on nucleation contemplates a much greater magnitude than what is proposed so far.

\section{Classic Theory of Nucleation}

In general the solidification occurs by a process of nucleation and growth in which the nuclei of the crystals are formed, which then by the addition of more atoms forms the 
grains or crystals. Nucleation occurs only when the kinetic energy of several atoms of the liquid metal reaches a value low enough to allow them to occupy equilibrium positions in the crystalline lattice, the conditions favoring the occurrence of nucleation depends on the thermodynamic aspects and the kinetic conditions of the transformation. When the solid is formed inside the liquid without the aid of any type of external energetic stimulant, it is said that the nucleation is homogeneous ${ }^{3}$.

In a phase transition such as solidification the transformation process cannot occur at any arbitrary undercooling. The reason is given by the high curvature of the interface associated with the crystal of atomic dimensions, this occurs because the difference of capillary pressure between the two phases, which is represented as curvature on the interface. This difference of pressure can be obtained by the Laplace-Young equation, which for a spherical geometry can be written as:

$\Delta p=2 \sigma K$

Where $\sigma$ is the surface energy density and $K$ is the average curvature of the interface. At constant pressure there is a single temperature at which a pure liquid substance can coexist in equilibrium with its solid phase, that is the phase transition temperature.

At this temperature the free energies of both phases are equal. Below the melting temperature $T_{f}$ the solid is the phase of lower energy, so this is the most stable one. But the liquid does not necessarily becomes solid immediately below the melting temperature, when an atomic cluster is arranged in a crystalline order to form an embryo a surface is formed, which carries a structure similar to both solid and the liquid phases, this surface separates the solid from the disordered liquid. Associated with this surface is a surface energy which is different from both the energy of the solid phase and the liquid phase. With the presence of the interface the equilibrium point of the system undergoes a temperature change given by $\Delta T_{r}$ due to the surface energy. The size of the crystal that allows the equilibrium between the curved crystal and the liquid is called critical size, it is given by a critical radius which in the case of spherical surfaces can be calculated considering the conditions of equilibrium between the crystal and the liquid ${ }^{1}$.

This condition suggests that homogeneous nucleation can occur through the formation of spherical embryos, since this geometric form is the one with the lowest surface/volume ratio $^{3}$. Under these conditions the total free energy change $\Delta G$ will be given by:

$\Delta G=\Delta G_{V}+\Delta G_{i}$

Where $\Delta G_{V}$ is the free energy variation associated with the volume of transformed material, and $\Delta G_{i}$ is the free energy variation associated with the created surface. The variation $\Delta G_{V}$ for a sphere of radius $r$ is given by:

$\Delta G_{V}=\frac{4}{3} \pi r^{3}\left(g_{S}-g_{L}\right)$
Where $g_{L}$ and $g_{S}$ are respectively the free energies per volume of the liquid and the solid. The variation of Gibbs free energy can be written as:

$$
G_{S}-G_{L}=\left(H_{S}-H_{L}\right)-T\left(S_{S}-S_{L}\right)
$$

Where $H$ is the enthalpy, $S$ is the entropy, $T$ is the transformation temperature and the subscripts $S$ and $L$ represents the solid and liquid phases. The enthalpy change is given by (4):

$d H=T d S+V d p$

Where $V$ is volume, and $p$ is pressure. Considering that the phase transition takes place under constant pressure, and integrating both sides of the Equation 5 it follows:

$H_{S}-H_{L}=Q_{l}$

Where $Q_{l}$ is the latent heat of fusion. The entropy change of the system is defined as $^{4}$ :

$d S=\frac{\delta Q r e v}{T}$

Where $Q_{\text {rev }}$ is the reversible heat exchanged in the transformation. Since during phase transition the temperature does not change, one can write the entropy variation as:

$\Delta S=\frac{Q_{l}}{T_{f}}$

Substituting Equations 6 and 8 into Equation 4 one gets:

$G_{S}-G_{L}=Q_{l}-T\left(\frac{Q_{l}}{T_{f}}\right)$

That can be written as:

$G_{S}-G_{L}=Q_{l}\left(1-\frac{T}{T_{f}}\right)$

And normalizing the Equation 10 per volume unit gives:

$g_{S}-g_{L}=L\left(1-\frac{T}{T_{f}}\right)$

Where $L$ is defined as latent heat of fusion per volume unit. And substituting Equation 11 into 3 gives:

$\Delta G_{V}=\frac{4}{3} \pi r^{3} L\left(1-\frac{T}{T_{f}}\right) \quad \Delta G_{V}=\frac{4}{3} \pi r^{3} L\left(\frac{T_{f}-T}{T_{f}}\right)$

Defining the undercooling $\Delta T$ as:

$\Delta T=T_{f}-T$

And substituting 13 into 12 gives: 
$\Delta G_{V}=\frac{4}{3} \pi r^{3} L \frac{\Delta T}{T_{f}}$

With the formation of the solid particle, a solid/liquid interface is established and consequently, additional energy associated with that interface. The free energy variation associated to this surface $\Delta G_{i}$, in the case of spherical geometry is given by:

$\Delta G_{i}=4 \pi r^{2} \sigma_{i}$

Where $\sigma_{i}$ is the surface energy density. Then the total free energy change in the formation of a solid spherical particle of radius $r$ is given by the sum of the two terms $\Delta G_{i}$ and $\Delta G_{V}$, that is, the sum of Equations 14 and 15:

$\Delta G=\frac{4}{3} \pi r^{3} L \frac{\Delta T}{T_{f}}+4 \pi r^{2} \sigma_{i}$

The sum of these two energy components leads to a resulting curve passing through a maximum value, which is defined as the activation energy for nucleation, which must be achieved to form a stable nucleus of critical radius. This point can be determined by making:

$$
\frac{d(\Delta G)}{d r}=0
$$

That is:

$$
\frac{4 \pi \Delta T L}{T_{f}} r_{c}^{2}+8 \pi \sigma_{i} r_{c}=0
$$

Where $r_{c}$ is the critical nucleus radius given by:

$$
r_{c}=\frac{2 \sigma_{i} T_{f}}{|L| \Delta T}
$$

And the maximum value of $\Delta G$ can be determined by substituting the critical radius of Equation 19 into Equation 16:

$$
\Delta G_{c}=\frac{16 \pi \sigma_{i}^{3}}{3\left(|L| \frac{\Delta T}{T_{f}}\right)^{2}}
$$

Particles that reach a radius greater than the critical radius become stable nuclei that will grow within the liquid phase, whereas those with a radius smaller than the critical radius return to the liquid state. Equations 19 and 20 clearly demonstrate the dependence of $r_{c}$ and $\Delta G_{c}$ with undercooling $\Delta T$, this undercooling is determined by the curvature of the interface.

In the following topics are proposed and discussed equations derived from thermodynamics that describes the effects of pressure to the critical radius and the activation energy.

\section{Pressure Influence on Activation Energy}

One of the main parameters of homogeneous nucleation is the critical radius of the nucleus, and the factors that determine it have essentially a thermodynamic nature being the main factor, according to the classical theory of nucleation, undercooling (temperature), but thermodynamically speaking pressure is a parameter as primitive as temperature, both intensive parameters and independent variables of Gibbs potential. Therefore considering a process to which the pressure variates, it must be taken into account throughout it.

As regards the literature that approaches this topic one can find a very straightforward review in Sobczak et al. ${ }^{2}$, where it can be concluded that according to the papers published so far, the effects of pressure on the critical radius of the nuclei are generally due to the variation in coexistence temperature and on the energy density of the interface.

In the classical theory of nucleation briefly exposed in this paper, it was discussed the thermodynamic relations that rules the process of homogeneous nucleation when there is no external pressure on the system. In this section the influence of external pressure on nucleation will be discussed considering it as an independent variable of the Gibbs potential as well as temperature, calculating its influence directly from the thermodynamic equations.

It was previously observed on equation 5 that the pressure differential reduces to zero in the case of constant pressure, but considering a pressure variation during nucleation, and considering the effect of pressure on the latent heat, the enthalpy change given would be calculated as:

$$
\Delta H=Q_{l p}+\int_{p_{0}}^{p} V d p
$$

Where $Q_{l p}$ is a pressure-dependent latent heat. Aluminum alloys are widely used in casting under pressure, so considering a system formed only by pure aluminum, one can calculate the value of the integral in Equation 21 by to writing $V$ as a $V(p)$ function. This can be done by using the state equation of ideal solids:

$V=V_{0}\left[1+\gamma\left(T-T_{0}\right)-\kappa\left(p-p_{0}\right)\right]$

Where $\gamma$ is the coefficient of volumetric expansion at constant pressure, defined as:

$\gamma=\left.\frac{1}{V_{0}} \frac{\partial \mathrm{V}}{\partial \mathrm{T}}\right|_{p=p_{0}}$

And $\kappa$ is the coefficient of isothermal compressibility, defined as:

$\kappa=-\left.\frac{1}{V_{0}} \frac{\partial \mathrm{V}}{\partial \mathrm{p}}\right|_{T=T_{0}}$

Now one must write $T$ as a $T(p)$ function in Equation 22. Observing the curve describing the dependence of the melting temperature as a function of the applied external pressure to a system formed of pure aluminum, presented in Jozsef and $\mathrm{Chen}^{5}$, it can be seen a linearity roughly between $0 G P a$ and $20 G P a$, as showed in Figure 1: 
This linearity is relevant because the vast majority of castings performed under pressure are within this range, in the industry it's generally in the order of $\approx 100 \mathrm{MPa}$. By restricting the range of allowable pressure variations to $0 \leq \triangle p \leq 10 G P a$, and considering a system made of pure aluminum, one can estimate a linear $T(p)$ function like:

$T_{f_{p}}=T_{f}+n\left(p-p_{0}\right)$

Where $T_{f_{p}}$ is the pressure-dependent fusion temperature, $T_{f}$ is the fusion temperature at atmospheric pressure, $n=\frac{d T}{d p}$ is the slope of this curve. In Sobczak et al. ${ }^{2}$, the value of this slope is presented as $53.7 \mathrm{~K} / \mathrm{GPa}$, as shown in Figure 2:

Now one can substitute Equation 25 into (22):

$$
\begin{aligned}
& V=V_{0}\left[1+\gamma n\left(p-p_{0}\right)-\kappa\left(p-p_{0}\right)\right] \\
& V=V_{0}\left[1+(\gamma n-\kappa)\left(p-p_{0}\right)\right]
\end{aligned}
$$

And use Equation 26 to calculate the integral of Equation 21:

$$
\begin{aligned}
& \int_{p_{0}}^{p} V_{0}\left[1+(\gamma n-\kappa)\left(p-p_{0}\right)\right] d p \\
& V_{0}\left[(\Delta p)+\frac{(\gamma n-\kappa)(\Delta p)^{2}}{2}\right]
\end{aligned}
$$

To quantify the constants of Equation 27, it should be remembered that this transformation takes place close to the melting point of aluminum, so it must be used the values of the quantities under these conditions. In Touloukian et al. ${ }^{6}$ one can find the coefficient of volumetric expansion as $\gamma=113,4 \times 10^{-6} K^{-1}$. With regard to the isothermal compressibility, it does not vary perceptibly between $300 \mathrm{~K}$ and $933 K$, as described in Hänström and Lazor $^{7}$, therefore it is reasonable to consider the value $\kappa=0,014 G P a^{-1}$ which is the one obtained at $300 \mathrm{~K}$.

By substituting the values of the constants in Equation 27, we obtain:

$V_{0}\left[(\Delta p)+\frac{\left(-0,0103 G P a^{-1}\right)(\Delta p)^{2}}{2}\right] \cong V_{0} \Delta p$

This shows that the second term on the right-hand side of Equation 27 is not relevant even at high pressures, becoming significant only at pressures in the order of $10 \mathrm{GPa}$ or greater. But since the pressure variations used in practical cases revolves around $100 \mathrm{MPa}$, it is reasonable to disconsider the second term of Equation 27.

Regarding the effect of pressure on the latent heat exchanged it can be given by the equation (29), as shown in ${ }^{8}$ :

$Q_{l p}=Q_{l}+\int_{T_{f}}^{T_{f p}}\left(c_{p(l)}-c_{p(s)}\right) d T$

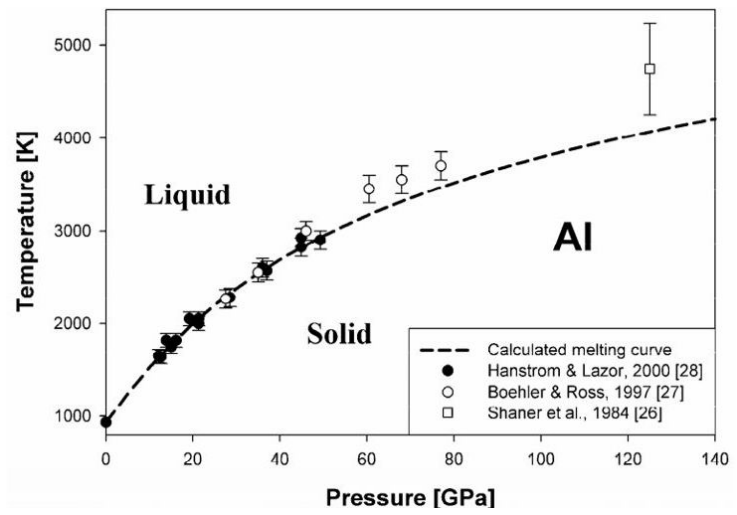

Figure 1. Coexistence curve for solid and liquid aluminum ${ }^{5}$.

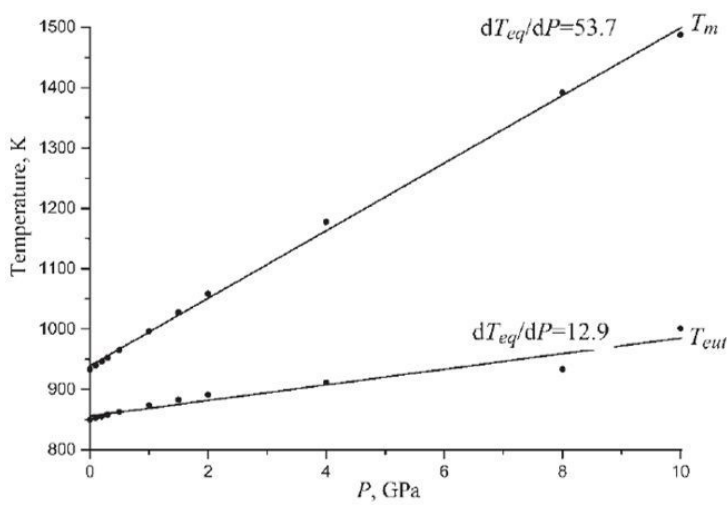

Figure 2. Melting point of aluminum and eutectic temperature of Al-Si system as function of pressure ${ }^{2}$.

Where $c_{p(l)}$ is the heat capacity of aluminium in the liquid phase, and $c_{p(s)}$ is the heat capacity of aluminium on the solid phase. The values the calorific capacities are given by ${ }^{9}$ :

$$
\begin{aligned}
& c_{p(l)}=7 J / \text { K.mol } \\
& c_{p(s)}=\left(4,94+2,96 \times 10^{-3} T\right) J / K . m o l
\end{aligned}
$$

Using the values of Equations 30 on the Equation 29 it gives:

$$
\begin{gathered}
Q_{l p}=Q_{l}+\int_{T_{f}}^{T_{f_{p}}}\left(7-4,94-2,96 \times 10^{-3} T\right) d T \\
Q_{l p}=Q_{l}+2,06 \Delta T-2,96 \times 10^{-3}\left(\frac{T_{f_{p}}{ }^{2}-T_{f}^{2}}{2}\right)
\end{gathered}
$$

Substituting Equation 25 on Equation 31 it can be determined the influence of pressure on the latent heat of fusion:

$$
Q_{l p}=Q_{l}+2,06 n \Delta p-\left(2,96 \times 10^{-3}\right)\left(\frac{(n \Delta p)\left(2 T_{f}+n \Delta p\right.}{2}\right)
$$

Using the values of the constants $T_{f}=933 \mathrm{~K}$ and $n=53,7 \mathrm{~K} / \mathrm{GPa}$, one can find that it takes about $8 G P a$ to 
decrease $5 \%$ the fusion latent heat, so at operational pressures this variation can be considered negligible. Therefore for this discussion onwards it will be considered that $Q_{l p}=Q_{l}$.

Given the considerations made regarding Equation 27 and (32), and substituting Equation 27 into (21) the variation of enthalpy is given by:

$\Delta H=Q_{l}+V_{0} \Delta p$

Considering the effect of the pressure on the entropy and substituting (25) into (7) gives:

$$
\Delta S=\frac{Q_{l}}{T_{f}+n \Delta p}
$$

Now substituting Equation 33 and Equation 34 in Equation 4 gives:

$$
\Delta G=\left(Q_{l}+V_{0} \Delta p\right)-\frac{T Q_{l}}{T_{f}+n \Delta p}
$$

Which can be written as:

$$
\begin{aligned}
& \Delta G=Q_{l}\left[1-\frac{T}{T_{f}+n \Delta p}\right]+V_{0} \Delta p \\
& \Delta G=Q_{l}\left[\frac{\Delta T+n \Delta p}{T_{f}+n \Delta p}\right]+V_{0} \Delta p
\end{aligned}
$$

Normalizing the Equation 36 per volume unit gives:

$g_{S}-g_{L}=L\left[\frac{\Delta T+n \Delta p}{T_{f}+n \Delta p}\right]+\Delta p$

Now Equation 37 can be substituted in (3) to obtain:

$$
\Delta G_{V}=\frac{4}{3} \pi r^{3}\left[\left(L\left(\frac{\Delta T+n \Delta p}{T_{f}+n \Delta p}\right)\right)+\Delta p\right]
$$

Taking into account the influence of pressure on the surface energy density, according to Sobczak et al. ${ }^{2}$ it is given by the following equation:

$\left(\frac{\partial \sigma}{\partial p}\right)_{T}=\left(\frac{V}{A}\right) \kappa \Delta p$

Where $A$ is the area of the nucleus. Considering a spherical nucleus, Equation 39 can also be written as a function of the radius:

$$
\left(\frac{\partial \sigma}{\partial p}\right)_{T}=\frac{\frac{4}{3} \pi r^{3}}{4 \pi r^{2}} \kappa \Delta p=\frac{r}{3} \kappa \Delta p
$$

Where $r$ is the radius of the nucleus, which suggests that the dependence of surface energy density on pressure is proportional to the size of the nucleus and the mechanical properties of the material. This equation was deduced assuming that the radius of the nucleus does not vary with the application of pressure.

In order to quantify the magnitude of the pressure effect on the density of surface energy, one can use typical values on Equation 40. Even without the effect of external pressure the critical radius of a stable nucleus assumes values in the order of $10^{-9} \mathrm{~m}^{1}, \kappa$ assumes a value of the order of $10^{-2} \mathrm{GPa}^{-1}$, and pressures used operationally are in the order of $10^{-1} \mathrm{GPa}$, so the variation of the energy density with pressure is in the order of:

$\left(\frac{\partial \sigma}{\partial p}\right)_{T}=10^{-12} \frac{\mathrm{J} / \mathrm{m}^{2}}{G P a}=10^{-8} \frac{\mathrm{J} / \mathrm{cm}^{2}}{G P a}$

Without external pressure the surface energy density takes values in the order of $10^{-6} \mathrm{~J}_{/ \mathrm{cm}^{2}}{ }^{10}$, so it will take at least $10 \mathrm{GPa}$ to make a relevant variation, therefore the effect of pressure on the density of energy will be disregarded in this discussion. Now replacing Equations 38 and (15) in (2) gives:

$$
\Delta G=\frac{4}{3} \pi r^{3}\left[\left(L\left(\frac{\Delta T+n \Delta p}{T_{f}+n \Delta p}\right)\right)+\Delta p\right]+4 \pi r^{2} \sigma_{i}
$$

Calculating the critical pressure-dependent radius gives:

$$
r_{c}^{p}=\frac{2 \sigma_{i}}{|L|\left(\frac{\Delta T+n \Delta p}{T_{f}+n \Delta p}\right)+\Delta p}
$$

Then, as can be seen from Equation 43, the increase in pressure causes a decrease in the critical radius. It can also be realized that at $\Delta p=0$ Equation 43 reduces to Equation 19. Now we can substitute Equation 43 in Equation 42 to obtain the pressure-dependent activation energy:

$$
\Delta G_{c}^{p}=\frac{16 \pi \sigma_{i}^{3}}{3\left(|L|\left(\frac{\Delta T+n \Delta p}{T_{f}+n \Delta p}\right)+\Delta p\right)^{2}}
$$

And again, as can be seen from Equation 44, the increase in pressure causes a decrease in the activation energy, just as it can be seen that at $\Delta p=0$ Equation 44 reduces to Equation 20 .

\section{Results and Discussions}

Comparing Equations 20 and (44), one observes that the pressure-dependent activation energy differs in two terms from the classical expression, highlighted in Figure 3:

$$
\Delta G_{c}^{p}=\frac{16 \pi \sigma_{i}^{3}}{3\left(|L|\left(\frac{\Delta T+n \Delta p}{T_{f}+n \Delta p}\right)+\Delta p\right)^{2}}
$$

Figure 3. Influence of pressure on activation energy.

The term in yellow showed in Figure 3 is caused by the change in the melting temperature, which is the only significant change caused by pressure according to previous papers found in literature. The term in red proposed on this paper, is accounted by a variation in enthalpy given by the change in the energy configuration, and obtained directly from its definition as showed previously. 
To grasp a quantitative notion of the effect of the term proposed on this paper, typical values of the quantities used on Equation 44 will be applied, it can be used the following values found in the literature: $T_{f}=933 \mathrm{~K}, \Delta T_{\max }=195 \mathrm{~K}$, $\sigma_{i}=120 \times 10^{-3} \mathrm{~J} / \mathrm{m}^{2}, L=1074 \times 10^{6} \mathrm{~J} / \mathrm{m}^{3^{10}}$, and $\triangle p=100 \mathrm{MPa}$, which is a typical pressure value used in solidification under external pressure. Substituting these values into the denominator of the equation shown in Figure 3, and comparing the magnitude of the term proposed in this paper with the expression proposed in literature we get:

$$
\begin{gathered}
|L|\left(\frac{\Delta T+n \Delta p}{T_{f}+n \Delta p}\right)-|L|\left(\frac{\Delta T}{T_{f}}\right)=4,86 \times 10^{6} \mathrm{~J} / \mathrm{m}^{3} \\
\left(|L|\left(\frac{\Delta T+n \Delta p}{T_{f}+n \Delta p}\right)+\Delta p\right)-|L|\left(\frac{\Delta T}{T_{f}}\right)=104,86 \times 10^{6} \mathrm{~J} / \mathrm{m}^{3}
\end{gathered}
$$

Figure 4. Comparison between the magnitude of the pressure effect proposed in the literature and that proposed in this work.

As can be seen in Figure 4 the difference caused by the effect of pressure on the phase coexistence temperature (in yellow) is on the order of $4,86 \times 10^{6} \mathrm{~J} / \mathrm{m}^{3}$, while the influence of pressure on enthalpy (in red) is given by $104,86 \times 10^{6} \mathrm{~J} / \mathrm{m}^{3}$, which is greater than its value by almost two orders of magnitude. Thus, the main influence of pressure on the activation energy is given by the change in the energetic state of the system due to its variation in enthalpy, and not due to the variation of the melting temperature as it is attributed in Sobczak et al. ${ }^{2}$.

Therefore for simplicity, the effect of the variation of the melting temperature in the activation energy will be neglected from this point onwards. Calculating the proportional variation of pressure in the activation energy we have:

$$
\eta \equiv \frac{\Delta G_{c}^{p}}{\Delta G_{c}}=\frac{3\left(|L|\left(\frac{\Delta T}{T_{f}}\right)+\Delta p\right)^{2}}{\frac{16 \pi \sigma_{i}^{3}}{3\left(|L|\left(\frac{\Delta T}{T_{f}}\right)\right)^{2}}}=\frac{\left(|L|\left(\frac{\Delta T}{T_{f}}\right)\right)^{2}}{\left(|L|\left(\frac{\Delta T}{T_{f}}\right)+\Delta p\right)^{2}}
$$

\section{Nucleus}

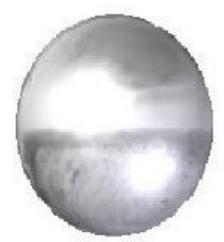

Substituting the values of the respective quantities on Equation 45 for the case of homogeneous nucleation gives:

$$
\eta=\frac{\Delta G_{c}^{p}}{\Delta G_{c}}=\frac{\left(1074\left(\frac{195}{933}\right)\right)^{2}}{\left(1074\left(\frac{195}{933}\right)+100\right)^{2}}=\left(\frac{224,46}{324,46}\right)^{2}=0,4785
$$

With the application of $100 \mathrm{MPa}$ the new activation energy corresponds to approximately $48 \%$ of its corresponding value to the same process without pressure, this considering an undercooling of $\Delta T=195 \mathrm{~K}$.

In real cases, even without pressure, nucleation is greatly facilitated by the presence of solid particles foreign to the liquid such as: impurities, the mold walls themselves and oxide layers formed in the portion of the liquid in contact with air, and others. In such cases, if the structure of the substrate is compatible with the liquid the activation energy is decreased due to geometric factors, decreasing the number of atoms of the critical nucleus, as well as the total surface tension imposed on the nucleus, as showed schematically in Figure 5. Due to the combination of these factors the activation energy decreases to a point where it can be reached even at much lower undercooling than the previously proposed, reaching typically values close to $10 \mathrm{~K}$. This mechanism is called heterogeneous nucleation and is the process that dominates nucleation in real solidifications, completely overlapping homogeneous nucleation since it is activated with much smaller undercooling. Although the presence of the substrate decreases the number of atoms in the critical nucleus, its radius does not vary in heterogeneous nucleation.

Similarly, the pressure also decreases the total energy of the stable nucleus by decreasing its number of atoms, but unlike the heterogeneous nucleation this decrease is caused by the reduction of the critical radius as showed in Equation 43. Being the proportional variation given by:

$\eta_{r}=\frac{r_{c}^{p}}{r_{c}}=\frac{|L|\left(\frac{\Delta T}{T_{f}}\right)}{|L|\left(\frac{\Delta T}{T_{f}}\right)+\Delta p}=\eta^{1 / 2}$

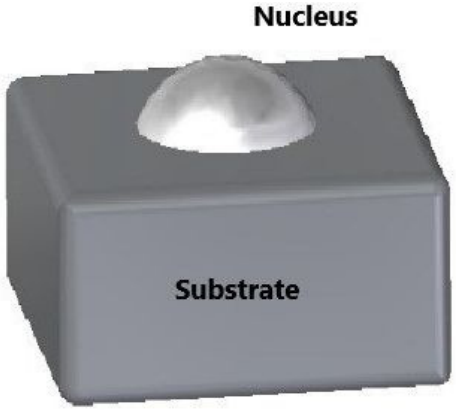

Heterogeneous nucleation

Figure 5. Schematic representation of heterogeneous and homogeneous nucleation. 

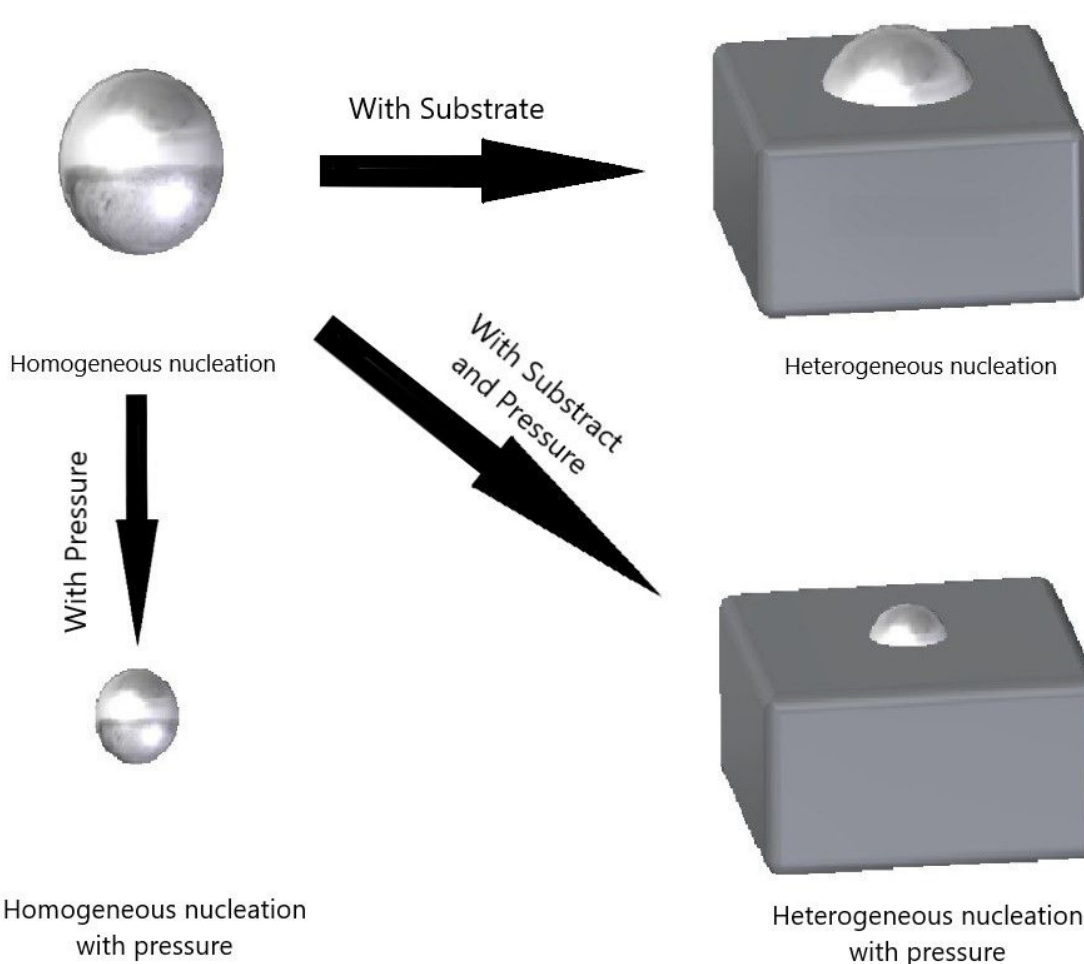

Heterogeneous nucleation
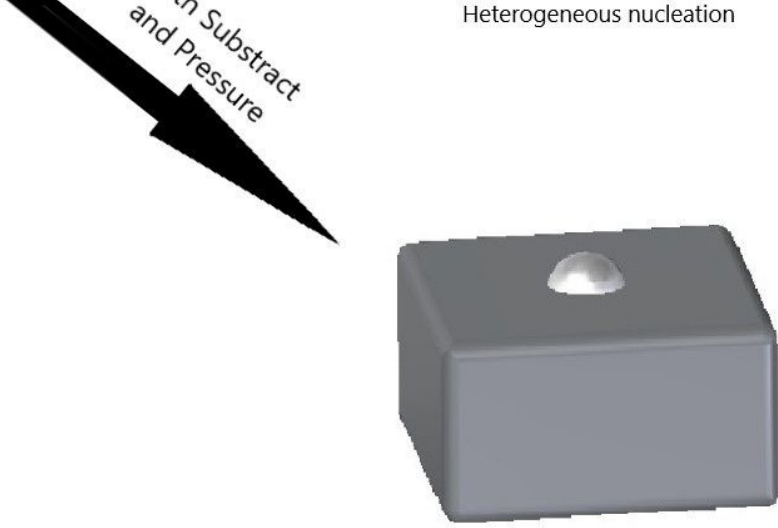

Heterogeneous nucleation with pressure

Figure 6. Schematic representation of the effect of pressure in combination with heterogeneous nucleation.

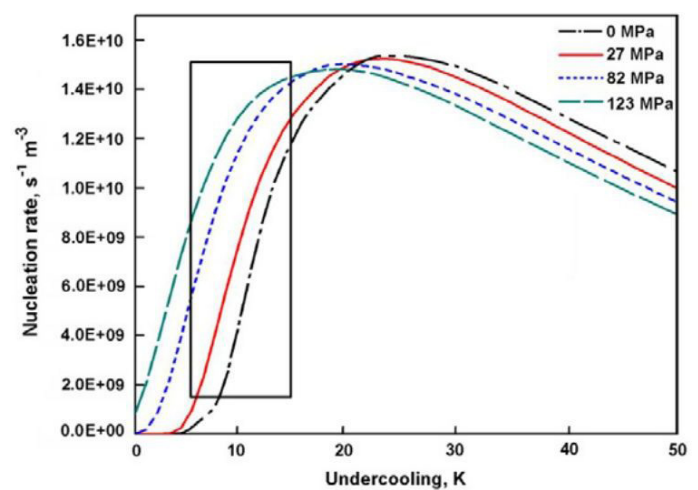

Figure 7. Influence of pressure on undercooling and nucleation rate of A356 aluminum alloy ${ }^{11}$.

Where $\eta_{r}$ is the proportional variation of the radius. In practical cases the two effects of heterogeneous nucleation and pressure combine, as shown schematically in Figure 6:

With application of external pressure, the activation energy decreases to an even lower value, compared to nucleation without pressure, allowing nucleation to begin with yet smaller undercooling. This effect predicted in this paper has already been reported in literature by Han et al. ${ }^{11}$, where was observed a decrease in undercooling corresponding to the maximum nucleation rate value, reproduced here in Figure 7:

Algebraically the effect shown on Figure 6 is described by the parameter $\eta$ given by Equation 45, which is strongly linked to the actual value of the undercooling, for example considering a real case of heterogeneous nucleation, taking an undercooling of $\Delta T=10 \mathrm{~K}$, a much smaller value is obtained for $\eta$ than obtained in Equation 46, on the order of 0,0106 , therefore $\eta$ is actually a function $\eta(\Delta p, \Delta T)$. This codependency between the effect of pressure and undercooling on the activation energy can be understood as a consequence of the Gibbs phase law given by ${ }^{4}$ :

$$
F=K-P+2
$$

Where $F$ is the number of freedom degrees of the system, $K$ is the number of component types and $P$ is the number of phases. Thus, considering that the system discussed is composed only of pure aluminum, applying the phase law it would have only one degree of freedom and, therefore, only one independent variable so that all the parameters that influence the energy state of the system must have links with each other. As the parameter $\eta$ is a dimensionless quantity the function $\eta(\Delta p, \Delta T)$ characterizes precisely this link between pressure and temperature. This co-dependence is seen in Figure 7, where it is observed that the increase in pressure causes a decrease in the undercooling corresponding to the maximum nucleation rate value, as previously discussed.

The Figure 8 shows the graph of the function $\eta\left(\Delta p, \Delta T_{0}\right)$, for a fixed undercooling $\Delta T_{0}=10 \mathrm{~K}$ in a pure aluminum system:

Observing the graph in Figure 8 considering the slope of the curve, one can see three distinct regions, first a region where the value of the parameter $\eta$ decreases rapidly (approximately between $0 M P a$ and $25 M P a$ ), then a saturation region where the slope of the curve is considerably reduced (between 25 MPa and $100 \mathrm{MPa}$ ), and then a third region where $\eta$ shows no appreciable variation with pressure (from $100 \mathrm{MPa}$ until the end of the chart). 


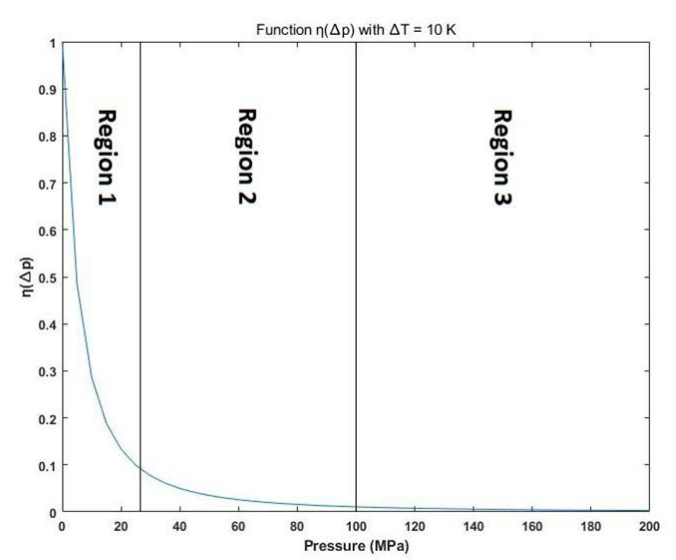

Figure 8. Typical curve of $\eta\left(\Delta p, \Delta T_{0}\right)$ according to Equation 45 , presenting 3 distinct regions.

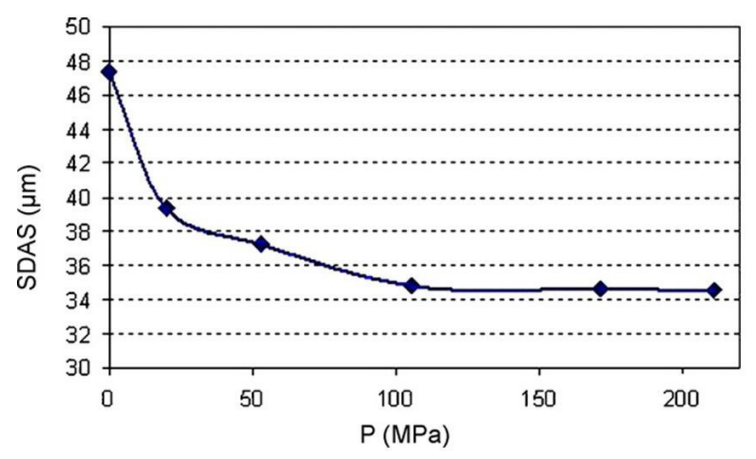

Figure 9. Secondary dendritic arm spacing of LM13 aluminum alloy ${ }^{12}$.
The decrease in nucleation activation energy is associated with the refining of structural parameters, in fact a behavior similar to that shown in the Figure 8 is reported in several studies in the literature regarding the influence of pressure on structural parameters, including at similar pressure ranges, as can be seen in Maleki et al. ${ }^{12}$, for LM13 aluminum alloy with casting temperature of $1003 K$ and mold temperature of $473 K$, it can be seen a considerable decrease in secondary dendritic arm spacing between $0 M P a$ and $25 M P a$, between $25 \mathrm{MPa}$ and $100 \mathrm{MPa}$ a saturation phase is observed, and after $100 \mathrm{MPa}$ it appears that the pressure no longer has a considerable refining effect, reproduced here in Figure 9:

In Obiekea et al. ${ }^{13}$ an A1350 alloy was solidified under pressure to analyze its effect on the mechanical properties and structural parameters of the alloy. Considering the conversion factor $1 \mathrm{Kgf} / \mathrm{cm}^{2}=0,098 \mathrm{MPa}$, a similar behavior is also observed, as there is a decrease in the slope of the curve describing the average grain diameter with increasing pressure from $40 \mathrm{MPa}$, reproduced here in Figure 10:

In Lima ${ }^{14}$ the effect of pressure on the average grain diameter in diluted alloys of the Al-Zn system, with 1\%,3\% and $5 \%$ zinc in its composition was investigated. The same tendency of decreasing the average diameter of the grain with applied pressure is observed, a more pronounced decrease until $50 \mathrm{MPa}$, between $50 \mathrm{MPa}$ and $100 \mathrm{MPa}$ a region of saturation is observed, and then from $100 \mathrm{MPa}$ until $150 \mathrm{MPa}$ pressure does not show an appreciable decrease in the average grain diameter, reproduced here in Figure 11:

In contrast to the model proposed in this paper, the influence of pressure on the activation energy described

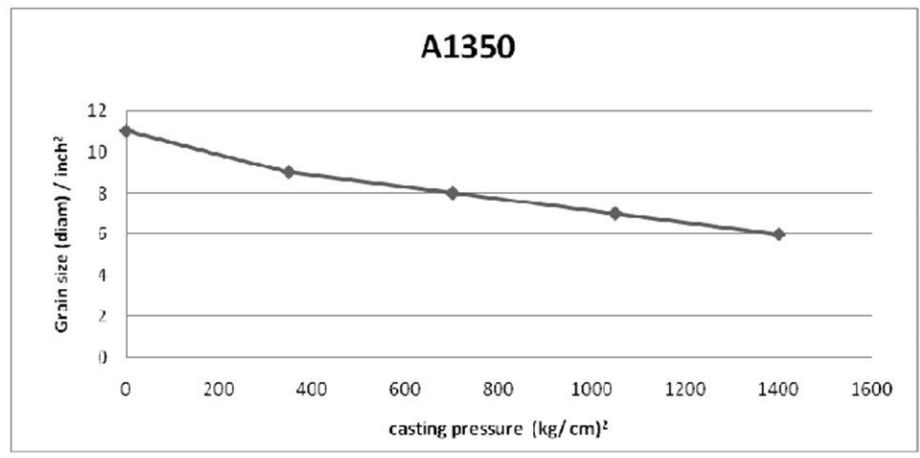

Figure 10. Average grain diameter as a function of pressure for A1350 aluminum alloy ${ }^{13}$.

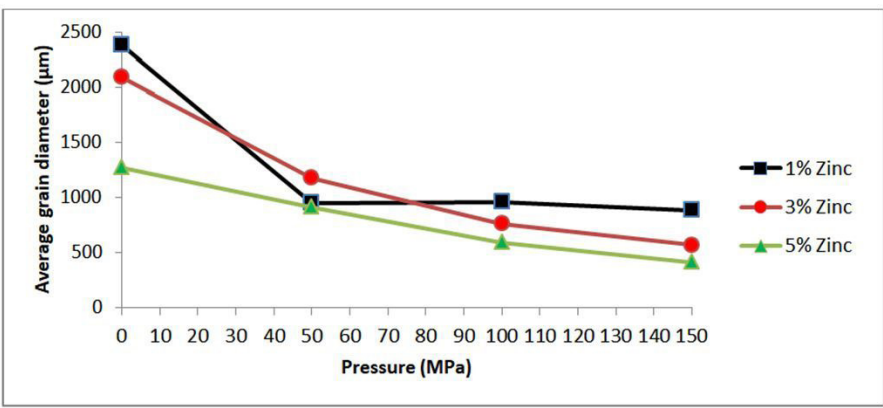

Figure 11. Average grain diameter versus pressure for aluminum alloys $\mathrm{Al}-\mathrm{Zn} 1 \%$, Al-Zn $3 \%$ and $\mathrm{Al}-\mathrm{Zn} 5 \%{ }^{14}$. 
in the literature so far can be represented by the yellow term in Figure 3, considering only the variation in the melting temperature the proportional term $\eta$ would be given by:

$$
\eta=\frac{\left(|L|\left(\frac{\Delta T}{T_{f}}\right)\right)^{2}}{\left(|L|\left(\frac{\Delta T+n \Delta p}{T_{f}+n \Delta p}\right)\right)^{2}}
$$

Which represents a hyperbole just like Figure 8, but the difference in order of magnitude between the yellow and red terms shown in Figure 4 implies that the saturation region, presented as region 2 in the Figure 8, would only be reached at pressures much higher than what is proposed by the Equation 45, and therefore would not be in agreement with the experimental results.

Of course the refining of structures does not depend only on the nucleation activation energy $\Delta G_{c}$, however the importance of this parameter is of remarkable significance, so much that it is clear that the influence of pressure on the structural parameters of aluminum alloys, can be seen as a reflection of the influence of pressure on activation energy, as can be observed by comparing the graph in Figure 8 with those in Figures 9, 10 and 11.

\section{Conclusion}

In conclusion the models used to describe the effects of pressure on nucleation published on literature so far do not fully contemplate it, so that in addition to the variation of the melting temperature, pressure still has a much more remarkable effect on the system energy state which is equivalent and co-dependent to undercooling. This effect causes a decrease in critical radius and consequently a decrease in activation energy acting in combination with the geometric effects of heterogeneous nucleation.

\section{Acknowledgments}

The authors acknowledge the Graduate Program in Mechanical Engineering of the Federal University of Paraiba (PPGEM), and the Coordination of Graduate Level Staff Improvement (CAPES).

\section{References}

1. Kurtz W, Fisher DJ. Fundamentals of solidification. Aedermannsdorf: Trans Tech Publications Ltd.; 1992.

2. Sobczak JJ, Drenchev L, Asthana R. Effect of pressure on solidification of metallic materials. Int J Cast Met Res. 2012;25(1):1-14.

3. Garcia A. Solidificação fundamentos e aplicações. Campinas: Editora da UNICAMP; 2011.

4. Greiner W, Neise L, Stöcker H. Thermodynamics and statistical mechanics. New York: Springer-Verlag; 1995.

5. Jozsef G, Chen J. Pressure effect on the melting temperature. arXiv. 2009;0906.3331.

6. Touloukian YS, Kirby RK, Taylor RE, Desai PD. Thermal expansion metallic elements and alloys. New York: Springer Science+Business Media; 1975 .

7. Hänström A, Lazor P. High pressure melting and equation of state of aluminium. J Alloys Compd. 2000;305(1-2):209-15.

8. Gaskell DR. Introduction to the thermodynamics of maeterials. New York: T\&F Books US; 2009.

9. Valencia JJ, Quested PN. ASM Handbook: casting. Material Park, OH: ASM International; 2008. p. 468-81. (vol. 15).

10. Campos MP, Davies GJ. Solidificação e fundição de metais e suas ligas. São Paulo: Editora da Universidade de São Paulo; 1978.

11. Han Z, Huang X, Luo AA, Sachdev AK, Liu B. A quantitative model for describing crystal nucleation in pressurized solidification during squeeze casting. Scr Mater. 2012;66(5):215-8.

12. Maleki A, Shafyei A, Niroumand B. Efect of squeeze casting parameters on microstructure of LM13 alloy. J Mater Process Technol. 2009;209(8):3790-7.

13. Obiekea K, Aku SY, Yawas DS. Influence of pressure on the mechanical properties and grain refinement of die cast aluminum A1350 alloy. Adv. Appl. Sci. Res. 2012;3(6):3663-73.

14. Lima RA. Influência de parâmetros operacionais na macroestrutura e propriedades mecânicas de ligas do sistema Al-Zn [dissertation]. João Pessoa: UFPB; 2014. 